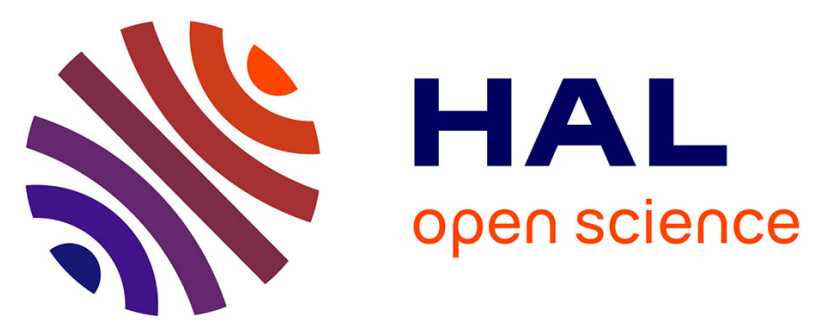

\title{
MicroRNAs facilitate skeletal muscle maintenance and metabolic suppression in hibernating brown bears
}

Bryan E. Luu, Etienne Lefai, Sylvain Giroud, Jon E. Swenson, Blandine

Chazarin, Guillemette Gauquelin-koch, Jon M. Arnemo, Alina L. Evans, Fabrice Bertile, Kenneth B. Storey

\section{To cite this version:}

Bryan E. Luu, Etienne Lefai, Sylvain Giroud, Jon E. Swenson, Blandine Chazarin, et al.. MicroRNAs facilitate skeletal muscle maintenance and metabolic suppression in hibernating brown bears. Journal of Cellular Physiology, 2020, 235 (4), pp.3984-3993. 10.1002/jcp.29294 · hal-02349938

\section{HAL Id: hal-02349938 \\ https://hal.science/hal-02349938}

Submitted on 9 Oct 2020

HAL is a multi-disciplinary open access archive for the deposit and dissemination of scientific research documents, whether they are published or not. The documents may come from teaching and research institutions in France or abroad, or from public or private research centers.
L'archive ouverte pluridisciplinaire HAL, est destinée au dépôt et à la diffusion de documents scientifiques de niveau recherche, publiés ou non, émanant des établissements d'enseignement et de recherche français ou étrangers, des laboratoires publics ou privés. 


\section{MicroRNAs facilitate skeletal muscle maintenance and metabolic suppression in hibernating brown bears}

Short title: MyomiRs in hibernating brown bears

Bryan E. Luu ${ }^{\mathrm{a}, \mathrm{b}}$, Etienne Lefai ${ }^{\mathrm{c}}$, Sylvain Giroud ${ }^{\mathrm{d}}$, Jon E. Swenson e,f, Blandine Chazarin ${ }^{\mathrm{g}, \mathrm{h}}$, Guillemette Gauquelin-Koch ${ }^{\mathrm{g}}$, Jon M. Arnemo ${ }^{\mathrm{i}, \mathrm{j}}$, Alina L. Evans ${ }^{\mathrm{i}}$, Fabrice Bertile ${ }^{\mathrm{h}}$, and Kenneth B. Storey a *

${ }^{a}$ Department of Biology, Carleton University, 1125 Colonel By Drive, Ottawa, K1S 5B6 Canada

${ }^{\mathrm{b}}$ Faculty of Medicine, McGill University, Montréal, Québec, H3G 0B1, Canada

c Université d'Auvergne, INRA, Unité de Nutrition Humaine, F-63000 Clermont-Ferrand, France

${ }^{\mathrm{d}}$ Research Institute of Wildlife Ecology, Department of Integrative Biology and Evolution, University of Veterinary Medicine, Vienna, Savoyenstraße 1, 1160 Vienna, Austria

${ }^{\mathrm{e}}$ Faculty of Environmental Sciences and Natural Resource Management, Norwegian University of Life Sciences, NO-1432 Ås, Norway

${ }^{f}$ Norwegian Institute for Nature Research, NO-7485 Trondheim, Norway

g Centre National d'Etudes Spatiales, CNES, F-75001 Paris, France

${ }^{\text {h }}$ Université de Strasbourg, CNRS, IPHC UMR 7178, F-670000 Strasbourg, France

${ }^{\text {i }}$ Department of Forestry and Wildlife Management, Inland Norway University of Applied Sciences, Campus Evenstad, NO-2480 Koppang, Norway

${ }^{\mathrm{j}}$ Department of Wildlife, Fish, and Environmental Studies, Swedish University of Agricultural Sciences, SE-901 83 Umeå, Sweden

\section{*Author for correspondence}

Dr. Kenneth B Storey

Carleton University

1125 Colonel By Drive, Ottawa, ON K1S 5B6

Tel: +1 613-520-2600, ext. 3678

Email: kenneth_storey@carleton.ca

ORCID: 0000-0002-7363-1853

\section{Author contributions}

All authors contributed to the conception and design of the project and to the editing of the manuscript. EL, SG, JES, BC, GG, JMA, ALE, and FB conducted the brown bear experiments and provided the tissue samples. BEL and KBS conducted biochemical assays. Data analysis and assembly of the manuscript was carried out by BEL, FB, and KBS. All authors read and approved the final manuscript.

\section{Data availability statement}

Research data are not shared. 


\section{Acknowledgements}

The authors thank J.M. Storey for editorial review of the manuscript. This work was supported by a Natural Sciences and Engineering Research Council of Canada (NSERC) Discovery grant (\#6793) to KBS, a French Space Agency (CNES) grant to FB (\#874), and CNRS and Strasbourg University grants to FB (projEX H2E and MyoBears project of the PEPS ExoMod program). KBS holds the Canada Research Chair in Molecular Physiology. BC was supported by a fellowship from the CNES and CNRS. The authors also thank the capture team from the Scandinavian Brown Bear Research Project (SBBRP), whose long-term funding has come primarily from the Swedish Environmental Protection Agency, the Norwegian Environment Agency, the Austrian Science Fund, and the Swedish Association for Hunting and Wildlife Management. This is paper $\mathrm{N}^{\circ} 283$ from the SBBRP (to be edited post-acceptance). The authors declare no conflicts of interest.

\section{Abstract}

Hibernating brown bears, Ursus arctos, undergo extended periods of inactivity and yet these large hibernators are resilient to muscle disuse atrophy. Physiological characteristics associated with atrophy resistance in bear muscle have been examined (e.g. muscle mechanics, neural activity) but roles for molecular signalling/regulatory mechanisms in the resistance to muscle wasting in bears still require investigation. Using RT-qPCR, the present study characterized the responses of 36 microRNAs linked with development, metabolism, and regeneration of skeletal muscle, in the vastus lateralis of brown bears comparing winter hibernating and summer active animals. Relative levels of mRNA of selected genes (mef $2 a$, pax7, id2, prkaal, and $m s t n$ ) implicated upstream and downstream of the microRNAs were examined. Results indicated that hibernation elicited a myogenic microRNA, or "myomiR", response via MEF2A-mediated signalling. Upregulation of MEF2A-controlled miR-1 and miR206 and respective downregulation of $\operatorname{pax} 7$ and $i d 2$ mRNA are suggestive of responses that promote skeletal muscle maintenance. Increased levels of metabolic microRNAs, such as miR27, miR-29, and miR-33, may facilitate metabolic suppression during hibernation via mechanisms that decrease glucose uptake and fatty acid oxidation. This study identified myomiR-mediated mechanisms for the promotion of muscle regeneration, suppression of ubiquitin ligases, and resistance to muscle atrophy during hibernation mediated by observed increases in miR-206, miR-221, miR-31, miR-23a, and miR-29b. This was further supported by the downregulation of myomiRs associated with muscle injury and inflammation (miR-199a and miR-223) during hibernation. The present study provides evidence of myomiR-mediated signalling pathways that are activated during hibernation to maintain skeletal muscle functionality in brown bears.

Keywords: atrophy; Mef2a; myomiR; noncoding RNA; ubiquitin ligase; Ursus arctos; 


\section{Introduction}

Hibernators are able to endure extreme environmental conditions that cannot be tolerated by most mammals. Typically, for small mammal hibernators, seasonal torpor is characterized by a prolonged state of inactivity, a strong decrease in core body temperature to near-ambient values, and the suppression of basal metabolic rate to levels often less than $10 \%$ of euthermic values (Heldmaier, Ortmann, \& Elvert, 2004; Jastroch et al., 2016; Ruf \& Geiser, 2015; Storey \& Storey, 2010). However, the iconic hibernators, bears (Ursidae), stand out from these others in several characteristics. Their large body mass makes it difficult to substantially decrease their body temperature such that core body temperature remains at $30-35^{\circ} \mathrm{C}$ during hibernation and they have the impressive ability to endure up to 6 months of inactivity without arousing and with no food or water intake, no urination or defecation, no muscle wasting, and no accumulation of nitrogen-containing waste products due to an active recycling program (Berg von Linde, Arevström, \& Fröbert, 2015). Previous studies of bears also showed no statistically significant changes in skeletal muscle fiber size between hibernating and non-hibernating animals (Hershey, Robbins, Nelson, \& Lin, 2008; Tinker, Harlow, \& Beck, 1998). In mammals, weight-bearing activities are crucial for the maintenance of skeletal muscle mass, morphology, and protein composition (Baldwin \& Haddad, 2001). Yet despite drastically reduced weight-bearing activity during hibernation, the skeletal muscle of bears retains most of its strength, with only a $23 \%$ decrease in maximal force (elicited by electrical stimulation) (Harlow, Lohuis, Beck, \& Iaizzo, 2001), whereas reduction in force and size as high as $60 \%$ is common in other non-hibernating mammals (Baldwin \& Haddad, 2001). In small hibernating mammals, up to 20-40\% of muscle protein content is lost at low body temperature (Steffen, Koebel, Musacchia, \& Milsom, 1991; Wickler, Hoyt, \& van Breukelen, 2017; Yacoe, 1983). However, hibernating bears lose only 4$11 \%$ of muscle protein content, and the size and number of their muscle fibres remain unchanged (Tinker et al., 1998).

In an attempt to better understand these observations, another study investigated the importance of the signalling pathways that underlie the resilience of skeletal muscle in hibernating brown bears (Ursus arctos). The authors challenged the concept that mechanical loading and neural activity of the muscles are the primary factors in maintaining skeletal muscle size (Lin, Hershey, Mattoon, \& Robbins, 2012). Indeed, studies on other non-hibernating mammals have demonstrated that regular neural activation or electrical stimulation of muscles is not enough to curtail muscle atrophy induced by mechanical unloading (Haddad, 2006; B. T. Zhang et al., 2010). Given that there is decreased muscle loading during hibernation, these authors studied the differences between the skeletal muscles (long distal extensor and cranial tibial) of hibernating and non-hibernating $U$. arctos that had undergone transections of the common peroneal nerve (Lin et al., 2012). Their results showed that active brown bears with denervation exhibited significantly higher muscle mass loss as compared to hibernating bears with denervation (Lin et al., 2012). The authors therefore suggested that a third factor is involved, proposing that differential regulation of signalling pathways, such as those that mediate 
the ubiquitin-mediated catabolism of contractile proteins, must be involved in atrophy resistance (Lin et al., 2012).

The observed resistance to muscle atrophy in hibernating bears could be elicited by signalling pathways that mediate changes in regulatory microRNAs. MicroRNAs are a class of highly conserved small non-coding RNAs that are capable of mediating the fate of mRNA transcripts in response to diverse signalling pathways in cells (Bartel, 2004; Storey \& Storey, 2010). As a component of RNA-induced silencing complexes, microRNAs contribute to posttranscriptional regulation by binding to complementary mRNAs to promote their degradation or inhibit their translation into functional proteins. MicroRNAs are differentially regulated in a number of small mammal hibernator models in a tissue specific manner, contributing to metabolic control, antioxidant defenses, and muscle maintenance networks during hibernation (Hadj-Moussa et al., 2016; Kornfeld, Biggar, \& Storey, 2012; Luu, Biggar, Wu, \& Storey, 2016; Storey \& Storey, 2010; Wu, Biggar, \& Storey, 2014) but, as yet, have not been evaluated in the context of bear hibernation. One direct regulator of skeletal muscle microRNA (myomiR) expression is the myocyte enhancer factor-2A (MEF2A) transcription factor, and its targets include miR-1, miR-133, and miR-206 (Liu et al., 2007; Rao, Kumar, Farkhondeh, Baskerville, \& Lodish, 2006). The MEF2A transcription factor is known to be crucial for skeletal muscle development, maintenance, and regeneration (Taylor \& Hughes, 2017). Previous studies have demonstrated that MEF2A signalling is activated in the skeletal muscle of thirteen-lined ground squirrels (Ictidomys tridecemlineatus) during hibernation and may contribute to muscle maintenance (Tessier \& Storey, 2010). Likewise, a study that profiled 10 microRNAs known to affect muscle-specific factors revealed that expression of these small non-coding transcripts are also differentially regulated in favour of skeletal muscle maintenance of hibernating bats (Myotis lucifugus) (Kornfeld et al., 2012).

The present study explores whether signalling pathways that impede muscle wasting are activated during hibernation in $U$. arctos. To address this, $U$. arctos mRNA and microRNA gene transcript sequences were obtained from available genomic data. RNA was isolated from skeletal muscle biopsies of 6 brown bears that were sampled during summer active and winter hibernating conditions. Specifically, mRNA transcript levels of MEF2A were measured by RTqPCR along with a group of 36 myomiRs that are known to control skeletal muscle regeneration, metabolism and/or be under the control of MEF2A (Diniz \& Wang, 2016; Horak, Novak, \& Bienertova-Vasku, 2016; Lima et al., 2017). Furthermore, selected downstream mRNA targets including myostatin ( $m s t n$ ), AMP-activated protein kinase (AMPK) catalytic subunit alpha 1 (prkaa1), paired box protein 7 (pax7), and inhibitor of DNA 2 (id2) that are known targets of the subset of microRNAs assessed herein were also quantified to better understand the potential roles of these microRNAs during bear hibernation. 
157

158

159

160

161

162

163

164

165

166

167

168

169

170

171

172

173

174

175

176

177

178

179

180

181

182

183

184

185

186

187

188

189

190

191

192

193

\section{Materials and Methods}

\section{$\underline{\text { Sampling of bear muscle }}$}

Six free-ranging brown bears (Ursus arctos; females; 2-3 years old), fitted with GPS collars, have been followed by the Scandinavian Brown Bear Research Project (SBBRP) in the Dalarna and Gävleborg counties, Sweden. Their immobilization was performed as previously reported (Evans et al., 2012), to allow sampling during hibernation in late February 2015 or 2016 and again during the active summer period in early June of the same years. After darting in their den (winter) or from a helicopter (summer), immobilized individuals were moved onto an insulated blanket prior to tissue sampling. Biopsies of the vastus lateralis muscle were collected and immediately frozen on dry ice before transfer to storage at $-80^{\circ} \mathrm{C}$ until analysis.

The mean body mass of hibernating bears $(46.8 \pm 8.7 \mathrm{~kg} ; 25-74 \mathrm{~kg})$ was not significantly different ( $\mathrm{p}=0.062$; paired $\mathrm{t}$-test) from that of the same bears when resampled as summer-active animals $(54.4 \pm 7.3 \mathrm{~kg} ; 37.8-83.6 \mathrm{~kg})$.

The study was approved by the Swedish Ethical Committee on Animal Experiment (applications \#C212/9, \#C47/9, \#C7/12, \#C268/12, and \#C18/15), the Swedish Environmental Protection Agency (NV-0758-14), and the Swedish Board of Agriculture (31-11102/12). All procedures complied with Swedish laws and regulations.

$\underline{\text { Total RNA isolation }}$

Total RNA was isolated from frozen tissue using TRIzol reagent (Invitrogen, Carlsbad, CA), as previously described (Luu, Green, Childers, Holahan, \& Storey, 2017). Briefly, samples of frozen bear muscle $(\sim 50 \mathrm{mg})$ were homogenized 1:20 w/v in TRIzol reagent and then combined with $300 \mu \mathrm{L}$ of chloroform. Samples were vigorously shaken by hand and incubated at room temperature for $5 \mathrm{~min}$ before centrifugation at $10,000 \mathrm{x} g$ for $15 \mathrm{~min}$ at $4^{\circ} \mathrm{C}$. The aqueous phase of each sample was then combined with $500 \mu \mathrm{L}$ isopropanol, incubated at room temperature for $15 \mathrm{~min}$, and then centrifuged at $12,000 \mathrm{x} g$ for $15 \mathrm{~min}$ at $4^{\circ} \mathrm{C}$ to facilitate RNA precipitation. RNA pellets were washed with $70 \%$ ethanol and air dried prior to resuspension in autoclaved RNase-free water. To standardize concentrations across samples, RNA concentration and purity (260/280 nm ratio) was assessed using a BioTek Take3 plate in a spectrophotometer (BioTek, Winooski, VT). Integrity of RNA was assessed by the appearance of $28 \mathrm{~S}$ and $18 \mathrm{~S}$ ribosomal RNA bands on a 1\% agarose gel stained with SYBR Green dye.

$\underline{\text { RNA polyadenylation and cDNA synthesis }}$

For analysis of microRNAs, samples of bear muscle RNA were polyadenylated and then cDNA synthesis was conducted as previously described (Biggar, Wu, \& Storey, 2014) using a Poly(A) polymerase tailing kit (Epicentre, Madison, WI) with a modified adapter primer (Supplementary Table S1) for use with short RNA molecules. 
Synthesis of cDNA was performed separately for mRNA quantification as previously described (Holahan, Smith, Luu, \& Storey, 2018). Standard oligo-dT (5'TTTTTTTTTTTTTTTTTTTTTTV-3'; where V=A, G, or C) was combined with RNA (1 $\mu \mathrm{g})$ in a total volume of $10 \mu \mathrm{L}$ of RNase-free water. Samples were incubated at $65^{\circ} \mathrm{C}$ for $5 \mathrm{~min}$ in an Eppendorf thermocycler, and then cooled on ice. Next, $4 \mu \mathrm{L}$ of $5 \mathrm{X}$ first strand buffer, $2 \mu \mathrm{L}$ of 0.1 M DTT, $1 \mu \mathrm{L}$ of $25 \mathrm{mM}$ dNTPs, and $1 \mu \mathrm{L}$ of MMLV reverse transcriptase enzyme (Invitrogen) were combined with the RNA/oligo-dT samples. Reactions were incubated at $42^{\circ} \mathrm{C}$ for $60 \mathrm{~min}$ in a thermocycler to facilitate cDNA synthesis.

\section{$\underline{\text { Primer design }}$}

MicroRNAs investigated in this study were selected from the literature based on their expression in skeletal muscle and their roles in regulating muscle cell processes (Diniz \& Wang, 2016; Horak et al., 2016; Lima et al., 2017). In order to determine the equivalent microRNA sequences in $U$. arctos, Mus musculus pre-microRNA sequences were obtained from miRBase (miRBase v.22; http://www.mirbase.org). Each M. musculus pre-microRNA sequence was aligned against the $U$. arctos WGS genome (SRA Accession No. SRX156136) using Sequence Read Archive Nucleotide BLAST (NCBI SRA BLAST; https://www.ncbi.nlm.nih.gov/sra). The conserved $U$. arctos pre-microRNA sequences were then aligned against $M$. musculus mature microRNA sequences from miRBase using Clustal Omega (EMBL-EBI; https://www.ebi.ac.uk/Tools/msa/clustalo/). From these alignments, the sequences of mature $U$. arctos microRNA were determined. Primers for relative microRNA quantification were designed as previously described (Biggar et al., 2014) and are listed in Supplementary Table S1.

Relative quantification of mRNA levels was performed for genes that are both upstream and downstream of microRNA regulatory pathways. Transcript levels of mef $2 a$, mstn, pax 7 , prkaal, and $i d 2$ were assessed. In order to design primers for these targets, the coding sequence of each gene was obtained from NCBI Gene (https://www.ncbi.nlm.nih.gov/gene/) for multiple mammals (U. maritimus, H. sapiens, M. musculus, S. scrofa, C. lupus, F. catus, O. aries, and E. caballus. These were aligned with Clustal Omega to determine highly conserved regions, for which primers were designed for gene expression quantification for U. arctos samples. Sequences deemed highly conserved and used for primer design had 100\% conservation with at least 4 different mammals. Primers were further analyzed with NCBI Primer-BLAST (https://www.ncbi.nlm.nih.gov/tools/primer-blast/) to demonstrate that they would amplify the target gene in multiple mammals. All mRNA primers used in this study are listed in

\section{Supplementary Table S1.}

\section{$\underline{\text { RT-qPCR }}$}

Relative quantification of gene transcripts used a BioRad CFX96 Connect System (BioRad, Hercules, CA). Reagents for RT-qPCR were prepared as previously described (Pellissier, Glogowski, Heinemann, Ballivet, \& Ossipow, 2006). Briefly, each amplification 
reaction included $2 \mu \mathrm{L}$ of $10 x$ buffer $(100 \mathrm{mM}$ Tris- $\mathrm{HCl}, \mathrm{pH} 8.5,500 \mathrm{mM} \mathrm{KCl}, 1.5 \%$ Triton $\mathrm{X}$ $100,20 \mathrm{mM} \mathrm{MgCl}_{2}$, and $2 \mathrm{mM}$ dNTPs), $6 \mu \mathrm{L}$ of $2 \mathrm{M}$ trehalose (BioShop, Canada), $0.5 \mu \mathrm{L}$ of $100 \%$ formamide (BioShop, Canada), $0.1 \mu \mathrm{L}$ of 1:100 SYBR green dye (Invitrogen), $1 \mu \mathrm{L}$ (1U) of Taq polymerase (BioShop, Canada), primers (synthesized by Integrated DNA Technologies, $250 \mathrm{nM}$ final concentration), and autoclaved water to a total volume of $20 \mu \mathrm{L}$. Each RT-qPCR reaction was analyzed with a melt curve analysis to ensure that the reaction was not amplifying more than one product and primers that amplified multiple products were not used for quantification. For relative quantification of microRNAs, reactions were brought to $95^{\circ} \mathrm{C}$ for 3 min, followed by 40 cycles of $95^{\circ} \mathrm{C}$ for $15 \mathrm{sec}$ and $60^{\circ} \mathrm{C}$ for $1 \mathrm{~min}$, as previously described (Biggar et al., 2014). Relative quantification of mRNAs used a modified protocol, where thermocycler conditions were 50 cycles of $95^{\circ} \mathrm{C}$ for $10 \mathrm{sec}, 57^{\circ} \mathrm{C}$ for $20 \mathrm{sec}$, and $72^{\circ} \mathrm{C}$ for $20 \mathrm{sec}$. Standard curves were performed with pooled cDNA samples and primer sets in order to assess the reaction specificity and efficiency for each gene transcript, and the efficiency of the primer pair was always used for quantification calculations.

\section{$\underline{\text { Statistical analyses }}$}

Since sampling of tissues was done in a paired manner where each bear was sampled (and tagged) in the winter and then sampled again the following summer, paired $t$-tests were used to determine statistically significant differences $(p<0.05)$ in microRNA and mRNA levels. Amplified transcripts were normalized against the expression of reference genes (U6 small nuclear RNA) for microRNA and gapdh for mRNA). The expression of these reference genes was determined to be stable between summer and winter hibernation conditions, as instructed (Schmittgen \& Livak, 2008). The comparative $\Delta \Delta \mathrm{Ct}$ method was used for relative expression calculations (Schmittgen \& Livak, 2008). Expression values for microRNA and mRNA were presented as mean \pm SEM with $n=6$ bears for microRNA analysis and $n=5$ bears for mRNA analysis). SigmaPlot v.12.5 was used for statistical analysis and bar graph generation (Systat Software Inc., San Jose, CA).

\section{Results}

Relative mRNA transcript levels of selected genes involved in the MEF2A signalling pathway were quantified via RT-qPCR to assess their transcriptional state in $U$. arctos vastus lateralis muscle during hibernation, as compared with the summer active state of the same individuals. Transcript levels of mef $2 a$ were $2.35 \pm 0.26$ fold higher during hibernation, relative to summer controls (Figure 1A). Seven microRNAs under MEF2A control (miR-1a-3p, miR-1a-15p, miR-1a-2-5p, miR-1b-5p, miR-133b-5p miR-133a-3p, and miR-206-3p) were also quantified. From this group, the levels of miR-1b-5p, miR-133a-3p, and miR-206-3p increased by $1.43 \pm 0.19,1.40 \pm 0.18$, and $1.65 \pm 0.17$ fold during hibernation, respectively (Figure 1B). The other four myomiRs were not responsive to hibernation. Two downstream targets of miR-1 and miR-206 were assessed with RT-qPCR to determine the potential impact of microRNA upregulation during hibernation (J.-F. Chen et al., 2010; Dey, Gagan, \& Dutta, 2011). Relative 
transcript levels of pax 7 and $i d 2$ decreased to $0.67 \pm 0.07$ and $0.40 \pm 0.06$ of summer active levels (Figure 1C).

Eighteen other microRNAs known for their regulatory roles in skeletal muscle (Lima et al., 2017) were also assessed in vastus lateralis muscle of active versus hibernating brown bears. Of these microRNAs, there were six that showed higher levels in hibernation. These were miR27b-5p, miR-27a/b-3p, miR-29a-5p, miR-29a-3p, miR-29b/c-3p, and miR-33-5p that showed significantly higher levels by $1.65 \pm 0.25,2.31 \pm 0.36,1.30 \pm 0.12,2.18 \pm 0.18,2.60 \pm 0.18$, and $1.24 \pm 0.08$ fold, respectively, in hibernating versus summer active bears (Figure 2A). To investigate the potential consequences of these increases in miR-33 (Davalos et al., 2011; Rottiers et al., 2011), relative levels of prkaal mRNA were quantified in bear muscle, but no changes were found between active and hibernating states (Figure 2B).

A subset of eleven myomiRs that are implicated in the atrophy and regeneration of skeletal muscles (Diniz \& Wang, 2016) were also assessed. Specifically, miR-23a-5p, miR23a/b-3p, miR-125b-3p, miR-199a-5p, miR-199a-3p, miR-221-3p, miR-222-5p, miR-222-3p, miR-223-5p, miR-223-3p, and miR-31-5p, were analyzed. Three microRNAs, miR-23a-5p, miR221-3p, and miR-31-5p were elevated during hibernation by $1.32 \pm 0.12,1.31 \pm 0.11$, and $1.26 \pm 0.09$ fold, respectively (Figure 3A). Oppositely, miR-199a-5p and miR-223-5p decreased to $0.66 \pm 0.04$ and $0.70 \pm 0.06$, respectively, of the levels in summer active bears (Figure 3A). Separately, transcripts of the myostatin gene, mstn, a mRNA target implicated in skeletal muscle maintenance (Miretti, Martignani, Accornero, \& Baratta, 2013) and a target of miR-27b (Figure 2A) displayed no changes between summer active and hibernation conditions (Figure 3B).

\section{Discussion}

Studies on brown bears (U. arctos) have suggested that the skeletal muscle resilience of these mammalian hibernators is determined by more than just mechanical loading and neural activity (Lin et al., 2012). A third factor proposed to contribute to the resilience of hibernator skeletal muscles is the modulation of signalling pathways, such as those that may be implicated in muscle catabolism. The present study explores muscle-specific cellular signalling pathways that are highly conserved in mammals. A group of 36 myomiRs known to be crucial for skeletal muscle development, metabolism, and regeneration (Diniz \& Wang, 2016; Horak et al., 2016; Lima et al., 2017) and 5 key upstream and downstream mRNA transcripts were investigated in vastus lateralis muscle from winter hibernating versus summer-active bears. The results reveal that microRNAs and mRNAs are differentially regulated in hibernating bears in a manner that would facilitate decreased metabolism, and maintenance of skeletal muscle over the winter months of torpor.

The observed higher levels of mef2a mRNA during hibernation suggest that, in a period when the bear is enduring prolonged inactivity without food or water intake (Berg von Linde et 
al., 2015), muscle mass (and strength) is still maintained; ie. little or no atrophy occurs (Figure 1A). Although analyses of MEF2A transcription factor activity were not performed in this study, higher mef $2 a$ mRNA (compared with summer) suggests that MEF2A protein abudance would also be higher during hibernation, which could result in more MEF2A-mediated gene transcription. Indeed, the MEF2A transcription factor is well known for its role in skeletal muscle development, maintenance, and regeneration (Taylor \& Hughes, 2017), and its upregulation at the mRNA and protein levels have been shown to facilitate skeletal muscle maintenance during hibernation in ground squirrels (Tessier \& Storey, 2010, 2012). Therefore, enhanced mef $2 a$ transcript levels may be an important underlying mechanism of skeletal muscle resilience in hibernating $U$. arctos.

Downstream targets of the MEF2A transcription factor were also assessed in order to elucidate the activated networks in skeletal muscles of bears during hibernation. MEF2A is known to directly regulate skeletal muscle microRNAs, which includes miR-1, -133, and -206 (Liu et al., 2007; Rao et al., 2006) and thereby has important roles in the regulation of skeletal muscle metabolism (Horak et al., 2016). The present study found that miR-1b-5p, miR-133a-3p, and miR-206-3p all increased during hibernation (Figure 1B), which is consistent with the observed increase of mef $2 a$ transcripts (leading to elevation of MEF2A protein). Downstream consequences on myogenic signalling pathways by miR-1 and miR-206 include downregulation of the pax7 gene, that encodes a transcription factor that is responsible for upregulating id 2 mRNA (J.-F. Chen et al., 2010; Dey et al., 2011). In turn, the ID2 protein is responsible for downregulating the myogenic transcription factor MYOD1 that, similarly to MEF2A, has a role in facilitating skeletal muscle growth and development through broad roles including the expression of miR-1 and miR-206 (Horak et al., 2016). Together, this suggests that activation of the MEF2A-mediated signalling pathway in hibernating bear skeletal muscle occurs through the upregulation of miR-1 and miR-206 and consequent downregulation of pax 7 and $i d 2$ (Figure $1 C)$.

Analyses of other microRNAs in hibernating $U$. arctos included a subset of fifteen microRNAs that target metabolic processes (Figure 2A) (Lima et al., 2017). Of these, six (miR27b-5p, miR-27a/b-3p, miR-29a-5p, miR-29a-3p, miR-29b/c, and miR-33-5p) were elevated during hibernation, compared with the summer active condition. The increases in miR-27, miR29 , and miR-33 found in hibernating bears suggest that these microRNAs may be potentially facilitating decreased glucose utilization. In a previous study, miR-27 action was shown to decrease glucose consumption when overexpressed in rat myoblast cell lines, and this was associated with decreased GLUT4 protein levels (Zhou et al., 2016). Indeed, when antisense sequences were introduced to suppress endogenous miR-27 activity in these cell lines, glucose consumption and uptake were enhanced and GLUT4 protein levels increased (Zhou et al., 2016). Thus, it can be postulated that elevated miR-27 in hibernating bears may be downregulating GLUT4 protein in skeletal muscle. In another study, a role for miR-29a/b/c in skeletal muscle glucose metabolism was indicated since these microRNAs were upregulated in a model of 
diabetic rats (He, Zhu, Gupta, Chang, \& Fang, 2007). Further experiments with cell lines showed that overexpression of miR-29a/b/c inhibited insulin-stimulated glucose uptake (He et al., 2007). These previous studies support the notion that increased miR-27 and miR-29 in hibernating bear muscle facilitate decreased glucose metabolism in part by reducing glucose uptake, which may also contribute to lowering basal metabolic rate.

Levels of prkaal mRNA (that encodes the catalytic subunit of the nutrient sensor AMPK) were also measured in hibernating $U$. arctos skeletal muscle since expression of this gene known to be downregulated by miR-33 (Davalos et al., 2011; Rottiers et al., 2011). Skeletal muscle AMPK has several regulatory roles in glucose and lipid metabolism, two of these being to increase glucose uptake and promote fatty acid oxidation as a means of enhancing ATP production under energy-stressed conditions (Collier, Bruce, Smith, Lopaschuk, \& Dyck, 2006; Jeon, 2016; O'Neill et al., 2014). As mentioned above, increases in miR-27, miR-29, and miR-33 in bear muscle may suggest that both glucose uptake and fatty acid oxidation are suppressed in hibernating bears. Thus, levels of muscle prkaal mRNA were assessed to determine how this downstream gene of microRNA action responded in hibernation. However, mRNA levels of prkaal did not change in muscle of hibernating bears, compared with summer active animals, suggesting that AMPK is not differentially regulated in summer vs winter, at least at the transcript level (Figure 2B). AMPK activity increased in some tissues of other hibernating species (e.g. in adipose of hibernating ground squirrels) during hibernation but was unchanged in other tissues including skeletal muscle (Horman, Hussain, Dilworth, Storey, \& Rider, 2005), an observation that is consistent with the present result for bears. It is important to note that the present study only assessed the mRNA levels of the catalytic subunit of AMPK in bear muscle, so further investigations are warranted to obtain a more complete understanding of the role of this energy/nutrient sensor in hibernating bears. It is still possible that hibernating bears are conserving energy by reducing both usage of glucose and fats. Recent studies have shown that enzymes involved in fatty acid oxidation are decreased in the skeletal muscle of hibernating bears, which is consistent with decreased energy expenditure (Chazarin et al., 2019). Overall, selected $U$. arctos myomiRs appear to be regulated in a manner that would facilitate the suppression of both glucose and lipid metabolism by targeting specific signalling pathways during hibernation.

The differentially expressed microRNAs identified in this study also have implications for skeletal muscle regeneration and integrity. In particular, miR-206, miR-221, and miR-31 that were elevated in hibernating bear muscle are known promoters of skeletal muscle regeneration (Figures 1B and 3A) (Diniz \& Wang, 2016; Greco et al., 2009; Togliatto et al., 2013). Two of the microRNAs that were reduced during hibernation, miR-199a and miR-223, are associated with inhibited myogenic differentiation, muscle disruption, injury, and inflammation (Figure 3A) (Alexander et al., 2013; Y. Chen, Melton, Gelfond, McManus, \& Shireman, 2012). Similarly to miR-27b and miR-29b (discussed above), miR-23a increases in hibernating bear muscle, and these three myomiRs not only increase in other species during hibernation (e.g. the little brown 
bat, Myotis lucifugus), but are also associated with muscle atrophy resistance, increased MEF2A activity, and decreased transcript levels of $m s t n$ that encodes myostatin, an inhibitor of muscle growth (Figure 2A and 3A) (Kornfeld et al., 2012; Miretti et al., 2013). In the present study, mstn mRNA levels were assessed in $U$. arctos skeletal muscle, but these did not change between summer active and hibernation conditions (Figure 3B). However, a lack of microRNA interaction with mstn mRNA cannot be ruled out since microRNAs function not only to facilitate mRNA degradation, but also manage mRNA storage, during which transcript levels can remain constant but their translation into protein is inhibited (Bartel, 2004). Indeed, mRNA storage appears to be a common feature of metabolic rate depression in multiple species and in response to a wide variety of environmental stress conditions, including hibernation (Tessier, Audas, $\mathrm{Wu}$, Lee, \& Storey, 2014; Tessier \& Storey, 2014). MiR-23a also promotes resistance to muscle atrophy by disrupting protein degradation. The mechanism by which this occurs is the direct suppression of two muscle-specific ubiquitin ligases, MAFbx/atrogin-1 and muscle RING-finger 1 , that are known to be induced during muscle atrophy and atrophy-associated protein degradation (Wada et al., 2011). Protein levels of ubiquitin ligases such as these are known to be modulated throughout the torpor-arousal cycle of hibernating ground squirrel hearts (Y. Zhang, Aguilar, \& Storey, 2016), and therefore, there is potential for them to participate in the skeletal muscle of bears. Together, the results from this study demonstrate how differential myomiR expression in hibernating $U$. arctos may help to regulate a diverse range of mechanisms that contribute to the bear's resilience to muscle atrophy.

An increase in miR-33 (discussed earlier for its potential role in regulating prkaal mRNA) and miR-29 in hibernation, as compared to active summer bears, may have a cytoprotective effect in the skeletal muscle of brown bears through the downregulation of sterol regulatory element-binding protein (SREBP). Overexpression of miR-33 in various cell lines have shown that it directly reduces the protein levels of SREBP-1, whereas miR-33 null mice had higher protein expression of SREBP-1 (Horie et al., 2013). It has also been shown that miR29 inhibits SREBP-1 expression in cancer cells (Ru et al., 2016). Although SREBP-1 has mostly been studied in the liver for its roles in metabolic regulation, studies have shown that it is significantly expressed in muscle cells (Ducluzeau et al., 2001; Guillet-Deniau et al., 2002), and furthermore, SREBP-1 nuclear protein content induces muscle atrophy in both in vitro and in vivo models (Lecomte et al., 2009). Many experiments have identified that SREBP regulates many myogenic and metabolic genes in skeletal muscle (Abiola et al., 2009; Lecomte et al., 2009; Rome et al., 2008). Of interest among SREBP targets are myogenic transcription factors MYOD1, MYOG, and MEF2C, which have shown to decrease with SREBP overexpression (Dessalle et al., 2012). Furthermore, SREBP is believed to induce skeletal muscle atrophy by leveraging the ubiquitin ligase system, in part by upregulating MuRF1 mRNA (Lecomte et al., 2009). The present results suggest that a similar effect might occur in bears, where downregulation of SREBP by miR-33 and/or miR-29 facilitates a cytoprotective effect in skeletal muscle. 


\section{Conclusion}

The present study sought to assess the role of microRNAs as regulatory factors in limiting atrophy of $U$. arctos skeletal muscle during winter hibernation. Analysis of selected muscle-specific microRNAs and mRNA transcripts indicated broad modulations occurring at both transcriptional levels, which were suggestive of post-transcriptional mechanisms activating myogenic pathways, suppressing glucose and lipid metabolism, and decreasing atrophic signalling during hibernation (Figure 4). The results support a hypothesis made previously that the modulation of cellular signalling pathways is crucial for the resilience of skeletal muscles against atrophy in hibernating brown bears (Lin et al., 2012). These current results warrant continuing investigation into other regulatory controls on signalling networks (such as SREBP and/or ubiquitin ligases) in order to further our understanding the complex molecular mechanisms involved in muscle maintenance in the hibernating brown bear.

\section{References}

Abiola, M., Favier, M., Christodoulou-Vafeiadou, E., Pichard, A. L., Martelly, I., \& GuilletDeniau, I. (2009). Activation of Wnt/ $\beta$-catenin signaling increases insulin sensitivity through a reciprocal regulation of Wnt10B and SREBP-1c in skeletal muscle cells. PLoS ONE, 4(12), e8509. https://doi.org/10.1371/journal.pone.0008509

Alexander, M. S., Kawahara, G., Motohashi, N., Casar, J. C., Eisenberg, I., Myers, J. A., ... Kunkel, L. M. (2013). MicroRNA-199a is induced in dystrophic muscle and affects WNT signaling, cell proliferation, and myogenic differentiation. Cell Death and Differentiation, 20(9), 1194-1208. https://doi.org/10.1038/cdd.2013.62

Baldwin, K. M., \& Haddad, F. (2001). Invited Review: Effects of different activity and inactivity paradigms on myosin heavy chain gene expression in striated muscle. Journal of Applied Physiology, 90(1), 345-357. https://doi.org/10.1152/jappl.2001.90.1.345

Bartel, D. P. (2004). MicroRNAs: Genomics, biogenesis, mechanism, and function. Cell, 116(2), 281-297. https://doi.org/10.1016/S0092-8674(04)00045-5

Berg von Linde, M., Arevström, L., \& Fröbert, O. (2015). Insights from the den: How hibernating bears may help us understand and treat human disease. Clinical and Translational Science, 8(5), 601-605. https://doi.org/10.1111/cts.12279

Biggar, K. K., Wu, C. W., \& Storey, K. B. (2014). High-throughput amplification of mature microRNAs in uncharacterized animal models using polyadenylated RNA and stem-loop reverse transcription polymerase chain reaction. Analytical Biochemistry, 462, 32-34. https://doi.org/10.1016/j.ab.2014.05.032

Chazarin, B., Storey, K. B., Ziemianin, A., Chanon, S., Plumel, M., Chery, I., ... Bertile, F. (2019). Metabolic reprogramming involving glycolysis in the hibernating brown bear skeletal muscle. Frontiers in Zoology, 16(1), 12. https://doi.org/10.1186/s12983-019-0312-2 
Chen, J.-F., Tao, Y., Li, J., Deng, Z., Yan, Z., Xiao, X., \& Wang, D.-Z. (2010). microRNA-1 and microRNA-206 regulate skeletal muscle satellite cell proliferation and differentiation by repressing Pax7. The Journal of Cell Biology, 190(5), 867-879. https://doi.org/10.1083/jcb.200911036

Chen, Y., Melton, D. W., Gelfond, J. A. L., McManus, L. M., \& Shireman, P. K. (2012). MiR351 transiently increases during muscle regeneration and promotes progenitor cell proliferation and survival upon differentiation. Physiological Genomics, 44(21), 10421051. https://doi.org/10.1152/physiolgenomics.00052.2012

Collier, C. A., Bruce, C. R., Smith, A. C., Lopaschuk, G., \& Dyck, D. J. (2006). Metformin counters the insulin-induced suppression of fatty acid oxidation and stimulation of triacylglycerol storage in rodent skeletal muscle. American Journal of PhysiologyEndocrinology and Metabolism, 291(1), E182-E189. https://doi.org/10.1152/ajpendo.00272.2005

Davalos, A., Goedeke, L., Smibert, P., Ramirez, C. M., Warrier, N. P., Andreo, U., ... Fernandez-Hernando, C. (2011). miR-33a/b contribute to the regulation of fatty acid metabolism and insulin signaling. Proceedings of the National Academy of Sciences, 108(22), 9232-9237. https://doi.org/10.1073/pnas.1102281108

Dessalle, K., Euthine, V., Chanon, S., Delarichaudy, J., Fujii, I., Rome, S., ... Lefai, E. (2012). SREBP-1 transcription factors regulate skeletal muscle cell size by controlling protein synthesis through myogenic regulatory factors. PLOS ONE, 7(11), e50878. https://doi.org/10.1371/journal.pone.0050878

Dey, B. K., Gagan, J., \& Dutta, A. (2011). miR-206 and -486 induce myoblast differentiation by downregulating Pax7. Molecular and Cellular Biology, 31(1), 203-214. https://doi.org/10.1128/MCB.01009-10

Diniz, G. P., \& Wang, D.-Z. (2016). Regulation of skeletal muscle by microRNAs. Comprehensive Physiology, 6(3), 1279-1294. https://doi.org/10.1002/cphy.c150041

Ducluzeau, P. H., Perretti, N., Laville, M., Andreelli, F., Vega, N., Riou, J. P., \& Vidal, H. (2001). Regulation by insulin of gene expression in human skeletal muscle and adipose tissue. Evidence for specific defects in type 2 diabetes. Diabetes, 50(5), 1134-1142. Retrieved from http://www.ncbi.nlm.nih.gov/pubmed/11334418

Evans, A. L., Sahlén, V., Støen, O. G., Fahlman, Å., Brunberg, S., Madslien, K., ... Arnemo, J. M. (2012). Capture, anesthesia, and disturbance of free-ranging brown bears (Ursus arctos) during hibernation. PLoS ONE, 7(7), e40520. https://doi.org/10.1371/journal.pone.0040520

Greco, S., De Simone, M., Colussi, C., Zaccagnini, G., Fasanaro, P., Pescatori, M., ... Martelli, F. (2009). Common micro-RNA signature in skeletal muscle damage and regeneration induced by Duchenne muscular dystrophy and acute ischemia. The FASEB Journal, 23(10), 3335-3346. https://doi.org/10.1096/fj.08-128579

Guillet-Deniau, I., Mieulet, V., Le Lay, S., Achouri, Y., Carré, D., Girard, J., ... Ferré, P. (2002). Sterol regulatory element binding protein-1c expression and action in rat muscles: insulin- 
like effects on the control of glycolytic and lipogenic enzymes and UCP3 gene expression. Diabetes, 51(6), 1722-1728. Retrieved from http://www.ncbi.nlm.nih.gov/pubmed/12031958

Haddad, F. (2006). Isometric resistance exercise fails to counteract skeletal muscle atrophy processes during the initial stages of unloading. Journal of Applied Physiology, 100(2), 433-441. https://doi.org/10.1152/japplphysiol.01203.2005

Hadj-Moussa, H., Moggridge, J. A., Luu, B. E., Quintero-Galvis, J. F., Gaitán-Espitia, J. D., Nespolo, R. F., \& Storey, K. B. (2016). The hibernating South American marsupial, Dromiciops gliroides, displays torpor-sensitive microRNA expression patterns. Scientific Reports, 6(1), 24627. https://doi.org/10.1038/srep24627

Harlow, H. J., Lohuis, T., Beck, T. D. I., \& Iaizzo, P. A. (2001). Muscle strength in overwintering bears. Nature, 409(6823), 997. https://doi.org/10.1038/35059165

He, A., Zhu, L., Gupta, N., Chang, Y., \& Fang, F. (2007). Overexpression of micro ribonucleic acid 29, highly up-regulated in diabetic rats, leads to insulin resistance in 3T3-L1 adipocytes. Molecular Endocrinology, 21(11), 2785-2794. https://doi.org/10.1210/me.2007-0167

Heldmaier, G., Ortmann, S., \& Elvert, R. (2004). Natural hypometabolism during hibernation and daily torpor in mammals. Respiratory Physiology and Neurobiology, 141(3), 317-329. https://doi.org/10.1016/j.resp.2004.03.014

Hershey, J. D., Robbins, C. T., Nelson, O. L., \& Lin, D. C. (2008). Minimal seasonal alterations in the skeletal muscle of captive brown bears. Physiological and Biochemical Zoology, 81(2), 138-147. https://doi.org/10.1086/524391

Holahan, M. R., Smith, C. A., Luu, B. E., \& Storey, K. B. (2018). Preadolescent phthalate (DEHP) exposure is associated with elevated locomotor activity and reward-related behavior and a reduced number of tyrosine hydroxylase positive neurons in post-adolescent male and female rats. Toxicological Sciences : An Official Journal of the Society of Toxicology, 165(2), 512-530. https://doi.org/https://dx.doi.org/10.1093/toxsci/kfy171

Horak, M., Novak, J., \& Bienertova-Vasku, J. (2016). Muscle-specific microRNAs in skeletal muscle development. Developmental Biology, 410(1), 1-13. https://doi.org/10.1016/j.ydbio.2015.12.013

Horie, T., Nishino, T., Baba, O., Kuwabara, Y., Nakao, T., Nishiga, M., ... Ono, K. (2013). MicroRNA-33 regulates sterol regulatory element-binding protein 1 expression in mice. Nature Communications, 4(1), 2883. https://doi.org/10.1038/ncomms3883

Horman, S., Hussain, N., Dilworth, S. M., Storey, K. B., \& Rider, M. H. (2005). Evaluation of the role of AMP-activated protein kinase and its downstream targets in mammalian hibernation. Comparative Biochemistry and Physiology - B Biochemistry and Molecular Biology, 142(4), 374-382. https://doi.org/10.1016/j.cbpb.2005.08.010

Jastroch, M., Giroud, S., Barrett, P., Geiser, F., Heldmaier, G., \& Herwig, A. (2016). Seasonal 
control of mammalian energy balance: Recent advances in the understanding of daily torpor and hibernation. Journal of Neuroendocrinology, 28(11). https://doi.org/10.1111/jne.12437

Jeon, S. M. (2016). Regulation and function of AMPK in physiology and diseases. Experimental \& Molecular Medicine, 48(7), e245. https://doi.org/10.1038/emm.2016.81

Kornfeld, S. F., Biggar, K. K., \& Storey, K. B. (2012). Differential expression of mature microRNAs involved in muscle maintenance of hibernating little brown bats, Myotis lucifugus: a model of muscle atrophy resistance. Genomics, Proteomics \& Bioinformatics, 10(5), 295-301. https://doi.org/10.1016/j.gpb.2012.09.001

Lecomte, V., Meugnier, E., Euthine, V., Durand, C., Freyssenet, D., Nemoz, G., ... Lefai, E. (2009). A New Role for Sterol Regulatory Element Binding Protein 1 Transcription Factors in the Regulation of Muscle Mass and Muscle Cell Differentiation. Molecular and Cellular Biology, 30(5), 1182-1198. https://doi.org/10.1128/mcb.00690-09

Lima, T. I., Araujo, H. N., Menezes, E. S., Sponton, C. H., Araújo, M. B., Bomfim, L. H. M., ... Silveira, L. R. (2017). Role of microRNAs on the regulation of mitochondrial biogenesis and insulin signaling in skeletal muscle. Journal of Cellular Physiology, 232(5), 958-966. https://doi.org/10.1002/jcp.25645

Lin, D. C., Hershey, J. D., Mattoon, J. S., \& Robbins, C. T. (2012). Skeletal muscles of hibernating brown bears are unusually resistant to effects of denervation. The Journal of Experimental Biology, 215(12), 2081-2087. https://doi.org/10.1242/jeb.066134

Liu, N., Williams, A. H., Kim, Y., McAnally, J., Bezprozvannaya, S., Sutherland, L. B., ... Olson, E. N. (2007). An intragenic MEF2-dependent enhancer directs muscle-specific expression of microRNAs 1 and 133. Proceedings of the National Academy of Sciences, 104(52), 20844-20849. https://doi.org/10.1073/pnas.0710558105

Luu, B. E., Biggar, K. K., Wu, C. W., \& Storey, K. B. (2016). Torpor-responsive expression of novel microRNA regulating metabolism and other cellular pathways in the thirteen-lined ground squirrel, Ictidomys tridecemlineatus. FEBS Letters, 590(20), 3574-3582. https://doi.org/10.1002/1873-3468.12435

Luu, B. E., Green, S. R., Childers, C. L., Holahan, M. R., \& Storey, K. B. (2017). The roles of hippocampal microRNAs in response to acute postnatal exposure to di(2-ethylhexyl) phthalate in female and male rats. NeuroToxicology, 59, 98-104. https://doi.org/10.1016/j.neuro.2017.02.004

Miretti, S., Martignani, E., Accornero, P., \& Baratta, M. (2013). Functional effect of mir-27b on myostatin expression: A relationship in piedmontese cattle with double-muscled phenotype. BMC Genomics, 14(1), 194. https://doi.org/10.1186/1471-2164-14-194

O’Neill, H. M., Lally, J. S., Galic, S., Thomas, M., Azizi, P. D., Fullerton, M. D., ... Steinberg, G. R. (2014). AMPK phosphorylation of ACC2 is required for skeletal muscle fatty acid oxidation and insulin sensitivity in mice. Diabetologia, 57(8), 1693-1702. https://doi.org/10.1007/s00125-014-3273-1 
Pellissier, F., Glogowski, C. M., Heinemann, S. F., Ballivet, M., \& Ossipow, V. (2006). Lab assembly of a low-cost, robust SYBR green buffer system for quantitative real-time polymerase chain reaction. Analytical Biochemistry, 350(2), 310-312. https://doi.org/10.1016/j.ab.2005.12.002

Rao, P. K., Kumar, R. M., Farkhondeh, M., Baskerville, S., \& Lodish, H. F. (2006). Myogenic factors that regulate expression of muscle-specific microRNAs. Proceedings of the National Academy of Sciences, 103(23), 8721-8726. https://doi.org/10.1073/pnas.0602831103

Rome, S., Lecomte, V., Meugnier, E., Rieusset, J., Debard, C., Euthine, V., ... Lefai, E. (2008). Microarray analyses of SREBP-1a and SREBP-1c target genes identify new regulatory pathways in muscle. Physiological Genomics, 34(3), 327-337. https://doi.org/10.1152/physiolgenomics.90211.2008

Rottiers, V., Najafi-Shoushtari, S. H., Kristo, F., Gurumurthy, S., Zhong, L., Li, Y., ... Näär, A. M. (2011). MicroRNAs in metabolism and metabolic diseases. Cold Spring Harbor Symposia on Quantitative Biology, 76, 225-233. https://doi.org/10.1101/sqb.2011.76.011049

Ru, P., Hu, P., Geng, F., Mo, X., Cheng, C., Yoo, J. Y., ... Guo, D. (2016). Feedback loop regulation of SCAP/SREBP-1 by miR-29 modulates EGFR signaling-driven glioblastoma growth. Cell Reports, 16(6), 1527-1535. https://doi.org/10.1016/j.celrep.2016.07.017

Ruf, T., \& Geiser, F. (2015). Daily torpor and hibernation in birds and mammals. Biological Reviews of the Cambridge Philosophical Society, 90(3), 891-926.

Schmittgen, T. D., \& Livak, K. J. (2008). Analyzing real-time PCR data by the comparative CT method. Nature Protocols, 3(6), 1101-1108. https://doi.org/10.1038/nprot.2008.73

Steffen, J. M., Koebel, D. A., Musacchia, X. J., \& Milsom, W. K. (1991). Morphometric and metabolic indices of disuse in muscles of hibernating ground squirrels. Comparative Biochemistry and Physiology -- Part B: Biochemistry And, 99(4), 815-819. https://doi.org/10.1016/0305-0491(91)90147-6

Storey, K. B., \& Storey, J. M. (2010). Metabolic rate depression: the biochemistry of mammalian hibernation. Advances in Clinical Chemistry, 52, 77-108. Retrieved from http://www.ncbi.nlm.nih.gov/pubmed/21275340

Taylor, M. V., \& Hughes, S. M. (2017). Mef2 and the skeletal muscle differentiation program. Seminars in Cell and Developmental Biology, 72, 33-44. https://doi.org/10.1016/j.semcdb.2017.11.020

Tessier, S. N., Audas, T. E., Wu, C.-W., Lee, S., \& Storey, K. B. (2014). The involvement of mRNA processing factors TIA-1, TIAR, and PABP-1 during mammalian hibernation. Cell Stress and Chaperones, 19(6), 813-825. https://doi.org/10.1007/s12192-014-0505-8

Tessier, S. N., \& Storey, K. B. (2010). Expression of myocyte enhancer factor-2 and downstream genes in ground squirrel skeletal muscle during hibernation. Molecular and Cellular Biochemistry, 344(1-2), 151-162. https://doi.org/10.1007/s11010-010-0538-y 
Tessier, S. N., \& Storey, K. B. (2012). Myocyte enhancer factor-2 and cardiac muscle gene expression during hibernation in thirteen-lined ground squirrels. Gene, 501(1), 8-16. https://doi.org/10.1016/j.gene.2012.04.004

Tessier, S. N., \& Storey, K. B. (2014). To be or not to be: the regulation of mRNA fate as a survival strategy during mammalian hibernation. Cell Stress and Chaperones, 19(6), 763776. https://doi.org/10.1007/s12192-014-0512-9

Tinker, D. B. B., Harlow, H. J. J., \& Beck, T. D. D. I. (1998). Protein use and muscle-fiber changes in free-ranging, hibernating black bears. Physiological and Biochemical Zoology, 71(4), 414-424. https://doi.org/10.1086/515429

Togliatto, G., Trombetta, A., Dentelli, P., Cotogni, P., Rosso, A., Tschöp, M. H., ... Brizzi, M. F. (2013). Unacylated ghrelin promotes skeletal muscle regeneration following hindlimb ischemia via SOD-2-mediated miR-221/222 expression. Journal of the American Heart Association, 2(6), e000376. https://doi.org/10.1161/JAHA.113.000376

Wada, S., Kato, Y., Okutsu, M., Miyaki, S., Suzuki, K., Yan, Z., ... Akimoto, T. (2011). Translational suppression of atrophic regulators by MicroRNA-23a integrates resistance to skeletal muscle atrophy. Journal of Biological Chemistry, 286(44), 38456-38465. https://doi.org/10.1074/jbc.M111.271270

Wickler, S. J., Hoyt, D. F., \& van Breukelen, F. (2017). Disuse atrophy in the hibernating golden-mantled ground squirrel, Spermophilus lateralis. American Journal of PhysiologyRegulatory, Integrative and Comparative Physiology, 261(5), R1214-R1217. https://doi.org/10.1152/ajpregu.1991.261.5.r1214

Wu, C.-W., Biggar, K. K., \& Storey, K. B. (2014). Expression profiling and structural characterization of microRNAs in adipose tissues of hibernating ground squirrels. Genomics, Proteomics \& Bioinformatics, 12(6), 284-291. https://doi.org/10.1016/j.gpb.2014.08.003

Yacoe, M. E. (1983). Protein metabolism in the pectoralis muscle and liver of hibernating bats, Eptesicus fuscus. Journal of Comparative Physiology B, 152(1), 137-144. https://doi.org/10.1007/BF00689738

Zhang, B. T., Yeung, S. S., Liu, Y., Wang, H. H., Wan, Y. M., Ling, S. K., ... Yeung, E. W. (2010). The effects of low frequency electrical stimulation on satellite cell activity in rat skeletal muscle during hindlimb suspension. BMC Cell Biology, 11(1), 87. https://doi.org/10.1186/1471-2121-11-87

Zhang, Y., Aguilar, O. A., \& Storey, K. B. (2016). Transcriptional activation of muscle atrophy promotes cardiac muscle remodeling during mammalian hibernation. PeerJ, 4, e2317. https://doi.org/10.7717/peerj.2317

Zhou, T., Meng, X., Che, H., Shen, N., Xiao, D., Song, X., ... Wang, L. (2016). Regulation of insulin resistance by multiple MiRNAs via targeting the GLUT4 signalling pathway. Cellular Physiology and Biochemistry, 38(5), 2063-2078. https://doi.org/10.1159/000445565 


\section{Figure legends}

653 Figure 1. MEF2A-controlled muscle microRNAs are upregulated in skeletal muscle of active 654 versus hibernating brown bears, $U$. arctos. (A) Relative transcript abundance of mef $2 a$ mRNA, 655 (B) Relative abundance of MEF2A-regulated microRNAs, and (C) Downstream mRNA targets 656 of miR-1 and miR-206, pax7 and id2 mRNA. MicroRNA and mRNA transcripts levels were standardized against the expression of $U 6$ and gapdh, respectively. Data are means \pm S.E.M., $n=$ 6 for microRNA and $n=5$ for mRNA individuals sampled in both winter hibernating and summer active states. Statistically significant differences are denoted with an asterisk $(*)$ and were analyzed with a paired $t$-test $(p<0.05)$.

661 Figure 2. Expression of metabolic microRNAs involved in glucose and lipid metabolism and downstream targets in skeletal muscle of hibernating brown bears, U. arctos. (A) Abundance levels of metabolic microRNAs, and (B) transcript levels of a downstream target, prkaal. MicroRNA and mRNA transcripts were standardized against the expression of U6 and gapdh, respectively. Data are means \pm S.E.M., $n=6$ for microRNA and $n=5$ for mRNA individuals sampled in both winter hibernating and summer active states. Statistically significant differences are denoted with an asterisk $(*)$ and were determined with a paired $t$-test $(p<0.05)$.

Figure 3. Expression of microRNAs linked with skeletal muscle regeneration and maintenance, and one downstream mRNA target, in skeletal muscle of the hibernating brown bear, U. arctos. (A) Relative abundance of microRNAs implicated in muscle maintenance during hibernation, and (B) transcript levels of the downstream target, mstn. MicroRNA and mRNA transcripts were standardized against the expression of $U 6$ and gapdh, respectively. Data are means \pm S.E.M., $n=$ 6 for microRNA and $n=5$ individuals sampled in both winter hibernating and summer active states. Statistically significant differences are denoted with an asterisk $(*)$ and were determined with a paired $t$-test $(p<0.05)$.

Figure 4. Proposed microRNA-mediated mechanism facilitating metabolic suppression and skeletal muscle maintenance in $U$. arctos during hibernation. Perforated boxes denote areas of the model supported by this study. Red font and asterisks denotes targets and areas warranting 
683 Supplementary Table S1. Primers for quantification of $U$. arctos mRNA and microRNA. 